ISSN 1991- 8690

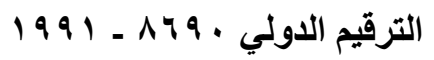

website : http:// jsci.utq.edu.iq

Email: utjsci@utq.edu.iq

\title{
Calculation of the Tunneling Current for Atom-sized Quantum Dot with Scanning Tunneling Microscope
}

\author{
Fouad Nimr Ajeel $^{1} \quad$ Jenan Majeed AL-Mukh $^{2}$ \\ ${ }^{1}$ Department of Physics- College of Science- University of Thi-Qar \\ ${ }^{2}$ Department of Physics- College of Education-University of Basra
}

\section{Abstract}

In this work, the tunneling current of adsorbed atom-sized quantum dot on metal surface with scanning tunneling microscope (STM) is calculated using nonequilibrium Green's function method of Keldysh. It is found that the correction to the tunneling current is expressed in terms of the transmission probability, where the correction is negative for the elastic part of the current and positive for the inelastic one. Depending on our calculations, we conclude that the position of electronic state of quantum dot, the vibrational mode energy, the strength of electron-vibration coupling and temperature, which important parameters to determine the physical features responsible for the processes concerned the nanostructure that are performed on the solid surface.

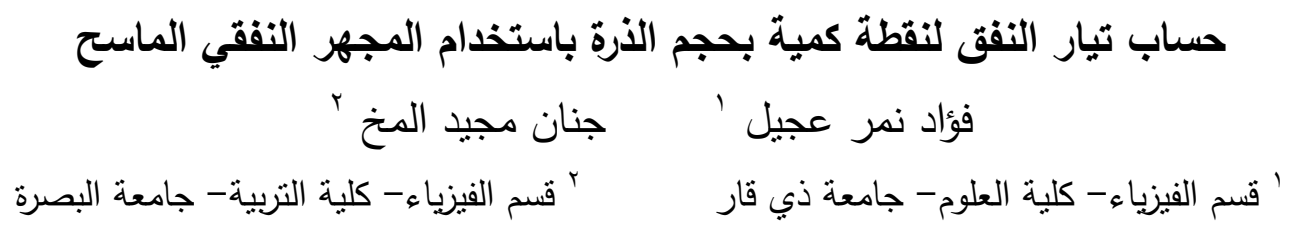

$\underline{\text { الخلاصة }}$

في هذا العمل، حسب تيار النفق لنقطة كمية بحجم الذرة ملتصقة على سطح معدن باستخدام المجهر النفقي الماسح (STM) استتاداً على طريقة دالة

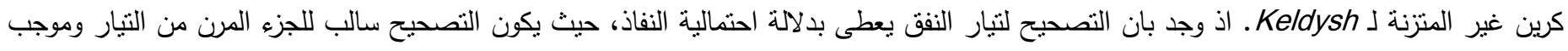

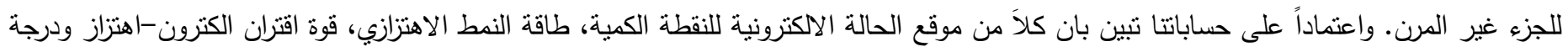
الحرارة كلها عوامل مهمة في تحديد المظاهر الفيزيائية المسؤولة عن العمليات المتعلقة بالتراكيب النانوية التي تتجز على التهابله السطح الصلب.

\section{Introduction}

One of the ultimate goals in Nanoscience is a realization of atomic and molecular scale devices. Scanning tunneling microscopy/spectroscopy (STM/STS) a decisive technique to study the structural and electronic properties of surfaces and adsorbates with atomic spatial ${ }^{[1]}$. Binnig et al. ${ }^{[2]}$ proposed, soon after the invention of STM ${ }^{[3]}$, a strategy to explore the vibrational properties of a single adsorbate based on the tunneling currents. This technique has opened a new avenue of single adsorbate vibrational spectroscopy to identify the vibrational "fingerprints" of the chemical reactions at surfaces. In addition to the identification of single adsorbed through vibrational fingerprint, it was demonstrated that the inelastic current may induce dynamical motions of adsorbate including rotation, dissociation, desorption and diffusion [4-6]. These 
current-induced dynamic processes may be important for a comprehensive understanding of the mechanism of a single adsorbate manipulation processes [7]. Many theoretical attempts have been made to elucidate the elementary properties involved in the tunneling currents of STM. Persson and Demuth [8] first discussed the inelastic tunneling using Bardeen's formula for electric current. Later, Persson and Baratoff [9] developed a theory of inelastic electron tunneling spectroscopy (IETS) using an adsorbate-induced resonance model, they predicted that the second derivative of the tunneling current with respect to a bias voltage exhibits a peak or dip depending on the energetic parameters of the system such as the position and broadening of the adsorbate induced resonance. Electron-vibration interaction effects in tunneling junctions were also studied by Caroli et al. [10], they derived analytical expressions for elastic and inelastic currents based on the nonequilibrium Green's function method and suggested a possible application to the internal vibrations of adsorbate molecules. The calculation of spatially resolved inelastic current was performed by Mingo and Makoshi [10] using a linear combination of atomic orbitals approach, and Lorente and Persson [12] using density functional treatment. However effects of vibrational damping rate on the inelastic current were neglected in both studies. A refinement of theory of the tunneling current of STM had been made by Tikhodeev et al. [13] using Anderson Hamiltonian supplemented with a phonon system using a nonequilibrium diagram technique $[14,15]$, they developed their theory to derive the elastic and inelastic currents and a relation between the inelastic fraction of tunneling current and the vibrational damping. They concluded that the total tunneling current either increases or decreases depending upon the opening of the inelastic tunneling channel.In this paper, we show that opening of the inelastic channel influences the total tunneling current, so that the total tunneling current at the threshold of the vibrational excitation $\mathrm{eV}=\hbar \Omega$ may change. Also, we show that there is a competition between the elastic and inelastic contributions to the tunneling current, where the total correction is negative for elastic current and positive for inelastic one. It is found that the positions of the quantum dot electronic state, bias voltage, the vibrational frequency, the strength of electron-vibration coupling and temperature, all are involved in IETprocesses i.e. all of them determine the spectral features of the tunneling current.

\section{Theory}

The total system consists of subsystems, a surface, a STM tip, a quantum dot (atom-sized) orbital is adsorbed on the surface and a quantum dot's phonon, whose energies (annihilation operators) are denoted by $E_{k}\left(C_{k}\right), E_{p}\left(C_{p}\right), E_{\text {dot }}\left(C_{\text {dot }}\right)$, and $\hbar \Omega(b)$

respectively. For simplicity, only a single quantum dot orbital and a single quantum dot phonon mode are taken into consideration. The Hamiltonian is given by $[9,13-$ 15]:

$$
\begin{aligned}
H & =\sum_{k} E_{k} C_{k}^{\dagger} C_{k}+\sum_{p} E_{p} c_{p}^{\dagger} C_{p}+E_{\text {dot }} C_{\text {dot }}^{\dagger} C_{\text {dot }}+\hbar \Omega\left(b^{\dagger} b+\frac{1}{2}\right) \\
& +\sum_{k}\left(V_{k \text { dot }} c_{k}^{\dagger} C_{\text {dot }}+\text { h.c. }\right)+\sum_{p}\left(V_{p d o t} C_{p}^{\dagger} C_{\text {dot }}+\text { h.c. }\right)+\chi C_{\text {dot }}^{\dagger} C_{\text {dot }}\left(b^{\dagger}+b\right)
\end{aligned}
$$

The tip and surface systems are assumed to be in thermal equilibrium at the temperature $\mathrm{T}$ and have independent chemical potentials $\mu_{\mathrm{t}}$ and $\mu_{\mathrm{s}}$ respectively. The difference corresponds to the bias voltage $\mathrm{eV}=$ $\mu_{\mathrm{s}}-\mu_{\mathrm{t}}$. The finite bias voltage is necessary for real vibrational excitations. The distribution function for electrons of the tip and surface subsystems, $n_{t}(E)$ and $\mathrm{n}_{\mathrm{s}}(\mathrm{E})$ are given by the Fermi distribution functions,

$$
n_{t, s}(E)=\left[\exp \left(\frac{E-\mu_{t, s}}{k_{B} T}\right)+1\right]^{-1}
$$

Electronic tunneling matrix elements $V_{k \text { dot }}$ (surfacequantum dot) and $V_{p \text { dot }}$ (tip-quantum dot) give rise to stationary current between the tip and the surface through the quantum dot orbital. Temporal population of electrons in the quantum dot orbital leads to the shift of the quantum dot equilibrium position. This process may induce dynamical motion of the quantum dot and open inelastic tunneling channels via the vibrational excitations and deexcitations. The electron-vibration coupling constant is denoted by $\chi$. The resonant widths caused by hybridization between the surface and the quantum dot (the tip and the quantum dot) are given by $\Delta_{\mathrm{S}}\left(\Delta_{\mathrm{t}}\right)$ :

$\Delta_{s, t}(E)=\pi \sum_{p, k}\left|V_{p(k) d o t}\right|^{2} \delta\left(E-E_{p, k}\right)=\Delta_{s, t}$

and the total resonant width is given by $\Delta=\Delta_{\mathrm{s}}+\Delta_{\mathrm{t}}$. For simplicity, these are assumed to be independent of energy i.e. we consider the wide-band limit [14].The nonequilibrium process of the electron tunneling between the tip and surface through the quantum dot level is described by the Nonequilibrium Green's Function (NEGF) Method [16,17]. This method 
describes the dynamical and statistical properties of the nonequilibrium system. So that we can find the occupation numbers and the spectra changes which are introduced into the system by the external action (the tip-surface bias voltage) mediated by the interactions in the system. NEGF method allows to go further and to find not only the nonequilibrium quantum dot population, but also the vibrational population [18]. The total stationary current $\mathrm{I}_{\mathrm{t}}(\mathrm{V})$ between the tip and surface through the quantum dot as a function of bias voltage in the stationary case (long after switching on of the bias voltage) is given by [18-20]:

$$
\begin{aligned}
& I_{t}(V)=\frac{2 e}{\hbar} \frac{\Delta_{s} \Delta_{t}}{\Delta} \int d E \rho_{\text {dot }}(E)\left[n_{s}(E)-n_{t}(E)\right] \\
& \text { where } \\
& \rho_{\text {dot }}(E)=-\frac{1}{\pi} \operatorname{Im}\left[E-E_{\text {dot }}-i \Delta(E)-\Sigma_{\text {dot }}^{\mathrm{r}}(E)\right]^{-1}
\end{aligned}
$$

$\rho_{\text {dot }}(E)$ is the full density of states (DOS) for electrons in quantum dot state. The imaginary part of the secondorder retarded self-energy for electrons $\Sigma_{\text {dot }}^{\mathrm{r}}(\mathrm{E})$ is given by:

$$
\begin{aligned}
\operatorname{Im} \sum_{\operatorname{dot}}^{\mathrm{r}}(E) & =\pi \chi^{2} \int d \omega \rho_{p h}(\omega) \times\left\{n_{p h}(\omega)\left[\rho_{\text {dot }}(E-\omega)+\rho_{\text {dot }}(E+\omega)\right]\right. \\
& \left.+\left[1-n_{\text {dot }}(E-\omega)\right] \rho_{\text {dot }}(E-\omega)+n_{\text {dot }}(E+\omega) \rho_{\text {dot }}(E+\omega)\right\}
\end{aligned}
$$

where $\rho_{\mathrm{ph}}(\omega)$ and $\mathrm{n}_{\mathrm{ph}}(\omega)$ are the vibrational density of states and the stationary distribution function for phonons, respectively:

$$
\begin{aligned}
\rho_{p h}(\omega) & =\frac{1}{\pi} \frac{\gamma_{\theta h}(\omega)}{(\omega-\hbar \Omega)^{2}+\gamma_{\theta h}^{2}(\omega)} \\
n_{p h}(\omega) & =\left[\exp \left(\frac{\hbar \omega}{k_{B} T}\right)-1\right]^{-1}+\frac{\pi \chi^{2}}{\gamma_{\theta h}(\omega)} \frac{\Delta_{s} \Delta_{t}}{\Delta^{2}} \int d E \rho_{d o t}(E) \rho_{d o t}(E+\omega) \\
& \times\left[n_{s}(E+\omega)-n_{t}(E+\omega)\right]\left[n_{s}(E)-n_{t}(E)\right]
\end{aligned}
$$

Eq. (8) includes the vibrational heating (in the second term) by inelastic tunneling electrons [21]. The vibrational damping rate $\gamma_{\mathrm{eh}}(\omega)$ due to electron-hole excitation is given by:

$$
\gamma_{\text {eh }}(\omega)=\pi \chi^{2} \int d E\left[n_{\text {dot }}(E)-n_{\text {dot }}(E+\omega)\right] \times \rho_{\text {dot }}(E) \rho_{\text {dot }}(E+\omega)
$$

where $\mathrm{n}_{\mathrm{dot}}(\mathrm{E})$ is the stationary distribution function for electrons,

$$
n_{\text {dot }}(E)=\frac{\Delta_{s} n_{s}(E)+\Delta_{t} n_{t}(E)}{\Delta}
$$

Since $\operatorname{Im} \Sigma_{\operatorname{dot}}^{\mathrm{r}}(\mathrm{E})$ is proportional to $\chi^{2}$, the total tunneling current can be decomposed into the terms of the zero and the second order of $\chi$. The elastic current in zeroth order is given by $[10,14,21]$ :

$$
I_{\theta l}^{(0)}=\frac{2 e}{\hbar} \frac{\Delta_{s} \Delta_{t}}{\Delta} \int d E \rho_{\text {dot }}(E)\left[n_{s}(E)-n_{t}(E)\right]
$$

While the additional components of the elastic and the inelastic currents in second order are, respectively, given by:

$$
\begin{aligned}
& I_{\theta l}^{(2)}=(-2 \pi) \frac{2 e}{\hbar} \frac{\Delta_{s} \Delta_{t}}{\Delta} \int d E\left(\rho_{\text {dot }}(E)\right)^{2} \operatorname{Im} \Sigma_{\text {dot }}^{\mathrm{r}}(E)\left[n_{s}(E)-n_{t}(E)\right] \\
& I_{\text {in }}^{(2)}=\frac{2 e}{\hbar} \frac{\Delta_{s} \Delta_{t}}{\Delta^{2}} \int d E \rho_{\text {dot }}(E) \operatorname{lm} \Sigma_{\mathrm{dot}}^{\mathrm{r}}(E)\left[n_{s}(E)-n_{t}(E)\right] \\
& \text { so that } \quad \quad \text { where } \quad I^{(2)}=I_{\theta l}^{(2)}+I_{\text {in }}^{(2)} \\
& I_{t}=I_{\theta l}^{(0)}+I^{(2)} \quad
\end{aligned}
$$

The elastic correction $\mathrm{I}_{\mathrm{el}}^{(2)}$ includes the process in which an electron is tunneling elastically via the quantum dot state from the surface to the tip emits and reabsorbs the phonon energy $\omega$ [10], it contributes to the inelastic current and not to the elastic current. While the inelastic contribution $\mathrm{I}_{\text {in }}^{(2)}$ corresponds to the tunneling process in which an electron for an occupied surface state is transported to an unoccupied tip state by emitting or absorbing the phonon energy . The inelastic contribution is always positive, while the elastic one gives always a reduction in the total tunneling current. As a result of this competition, the influence of the electron-vibration interaction on the elastic current $\mathrm{I}_{\mathrm{el}}^{(0)}$ is given by the second order correction to the total current,

$$
I^{(2)}=\frac{2 e}{\hbar} \frac{\Delta_{s} \Delta_{t}}{\Delta^{2}} \int d E \rho_{\text {dot }}(E)\left[1-2 \pi \Delta \rho_{\text {dot }}(E)\right] \operatorname{Im} \sum_{\text {dot }}^{\mathrm{r}}(E)\left[n_{s}(E)-n_{t}(E)\right]
$$

which becomes positive or negative depending on the sign of $F(E)$,

$$
F(E)=1-2 \pi \Delta \rho_{\text {dot }}(E)=1-\frac{\Delta^{2}}{2 \Delta_{s} \Delta_{t}} T(E)
$$

and,

$$
T(E)=4 \pi \frac{\Delta_{s} \Delta_{t}}{\Delta} \rho_{\text {dot }}(E)
$$

where $\mathrm{T}(\mathrm{E})$ is the transmission probability [22].Usually, the STM tip is made of tungsten while the adsorbate and the surface is made in accordance with 
the objective and purpose for which the measurement takes place. In this study the surface used is clean $\mathrm{Cu}$ (100) with adsorbed atom-sized quantum dot. The quantum dot is taken as a potential box with rigid walls where energy states depend on the dimensions of the box $\left(\mathrm{L}_{\mathrm{x}}, \mathrm{L}_{\mathrm{y}}, \mathrm{L}_{\mathrm{z}}\right)$. For a cubic potential box and nondegenerate state $\mathrm{n}_{\mathrm{x}}=\mathrm{n}_{\mathrm{y}}=\mathrm{n}_{\mathrm{z}}=1$, the energy states is given by the following relationship [23]:

$E_{111}\left(L_{\text {dot }}\right)=\frac{3 \pi^{2} \hbar^{2}}{2 m^{*}}\left(\frac{1}{L_{\text {dot }}^{2}}\right)$

$\mathrm{L}_{\text {dot }}$ represents the side length of the cube where $\mathrm{L}_{\mathrm{dot}}=\mathrm{L}_{\mathrm{x}}=\mathrm{L}_{\mathrm{y}}=\mathrm{L}_{\mathrm{z}}$ and $\mathrm{m}^{*}$ represent the effective mass with $\mathrm{m}^{*}=0.06 \mathrm{~m}_{\mathrm{e}}$, and $\mathrm{m}_{\mathrm{e}}$ represents the mass of the electron.

\section{Numerical Calculation}

A numerical result is performed to: (i) calculate the tunneling currents. (ii) study the competition between the elastic and inelastic correction to the total tunneling current. (iii) study how the tunneling currents are change as a function of all the treatment's variables especially the position of the quantum dot electronic state $E_{\mathrm{dot}}$, the bias voltage $\mathrm{eV}$, the vibrational frequency $\Omega$, the electron-vibration coupling $\chi$ and the temperature $\mathrm{T}$ at which the experiment is performed. The tunneling currents calculations for the resonance case is only presented. Fig. 1 shows $\mathrm{I}_{\mathrm{el}}^{(0)}, \mathrm{I}^{(2)}$ and $\mathrm{I}_{\mathrm{t}}$ that are calculated numerically by using Eqs. (11), (15) and (14) respectively, for resonance case $E_{\text {dot }}=0.0 \mathrm{meV}$ with $\mathrm{T}=15 \mathrm{~K}, \Delta_{\mathrm{t}}=5 \mathrm{meV}, \Delta_{\mathrm{s}}=1000 \mathrm{meV}$ and $\hbar \Omega=50 \mathrm{meV}$. The chemical potential of the surface $\mathrm{E}_{\mathrm{F}}$ is chosen as the energy reference. It is found that $\mathrm{I}_{\mathrm{el}}^{(0)}$ is independent of the electron-vibration coupling (see Fig. 1a). while $\mathrm{I}^{(2)}$ decreases with rise of the electronvibration coupling strength (see Fig. 1b) when $\mathrm{eV} \geq \hbar \Omega$ i.e. the inelastic channel is opened. This inelastic current adds to the elastic one, so that the total current $I_{t}$ changes. $I_{t}$ is weakly dependent on the electronvibration coupling (see Fig. 1c), as a result $\mathrm{I}_{\mathrm{el}}^{(0)}$ contribution to $I_{t}$ is always greater. Fig. 2 , shows the dependence of the tunneling current on the vibrational frequency. The same previous remarks are concluded for $\mathrm{I}_{\mathrm{el}}^{(0)}, \mathrm{I}^{(2)}$ and $\mathrm{I}_{\mathrm{t}}$ that are shown in Fig. 1.In Fig. 3 , we check the dependence of tunneling currents on temperature. It is clear from this figure that $\mathrm{I}_{\mathrm{el}}^{(0)}, \mathrm{I}^{(2)}$ and $I_{t}$ are approximately independent on temperature, but this conclusion is not accurate unless one knows the behavior of $\left(-\mathrm{I}_{\mathrm{el}}^{(2)}\right)$ and $\left(\mathrm{I}_{\mathrm{in}}^{(2)}\right)$ with temperature and other variables mentioned above. Fig. 4 shows the dependence of the elastic contribution $\left(-\mathrm{I}_{\mathrm{el}}^{(2)}\right)$ and the inelastic contribution $\left(\mathrm{I}_{\mathrm{in}}^{(2)}\right)$ on the previous parameters, It is found that:

1. $\left|\mathrm{I}_{\mathrm{el}}^{(2)}\right|=\mathrm{I}_{\mathrm{in}}^{(2)}$ for $|\mathrm{eV}|<\hbar \Omega$.

2. $\left|\mathrm{I}_{\mathrm{el}}^{(2)}\right|>\mathrm{I}_{\text {in }}^{(2)}$ for $|\mathrm{eV}|>\hbar \Omega$, leading to the negative correction to the total current.

The gradual increase in both $\left|\mathrm{I}_{\mathrm{el}}^{(2)}\right|$ and $\mathrm{I}_{\text {in }}^{(2)}$ below the threshold at $\mathrm{eV}=\hbar \Omega$ is due to the broadening of the vibrational density of states. The elastic $\left|\mathrm{I}_{\mathrm{el}}^{(2)}\right|$ and inelastic $\mathrm{I}_{\text {in }}^{(2)}$ correction decrease with rise of the vibrational frequency.

\section{Conclusions}

Depending on our results, the following conclusions are reported:

1- When the energy of tunneling electrons is smaller than the vibrational energy $\hbar \Omega$ of the adsorbate, no inelastic tunneling occurs. When $V \geq \hbar \Omega$, the inelastic channel becomes allowed.

2- An adsorbate overcomes the potential barrier by vibrational activation through a competition between gaining energy from the tunneling current and losing energy to the electron-hole pair excitation.

3 - The vibrational activation energy will be always dominant especially at $\mathrm{V}>\hbar \Omega$.

4- Usually, at low temperatures where $\mathrm{k}_{\mathrm{B}} \mathrm{T} \ll \hbar \Omega$, the vibrational broadening is neglected. But it is taken into consideration in our calculations.

5- The inelastic contribution is always positive, while the elastic contribution causes always a reduction in the total current. Therefore, the comparison between the values of the contributions $\mathrm{I}_{\mathrm{el}}^{(2)}$ and $\mathrm{I}_{\mathrm{in}}^{(2)}$ for various values of parameters is very important because it gives an idea about the emission or absorption process to excite a vibrational mode. 


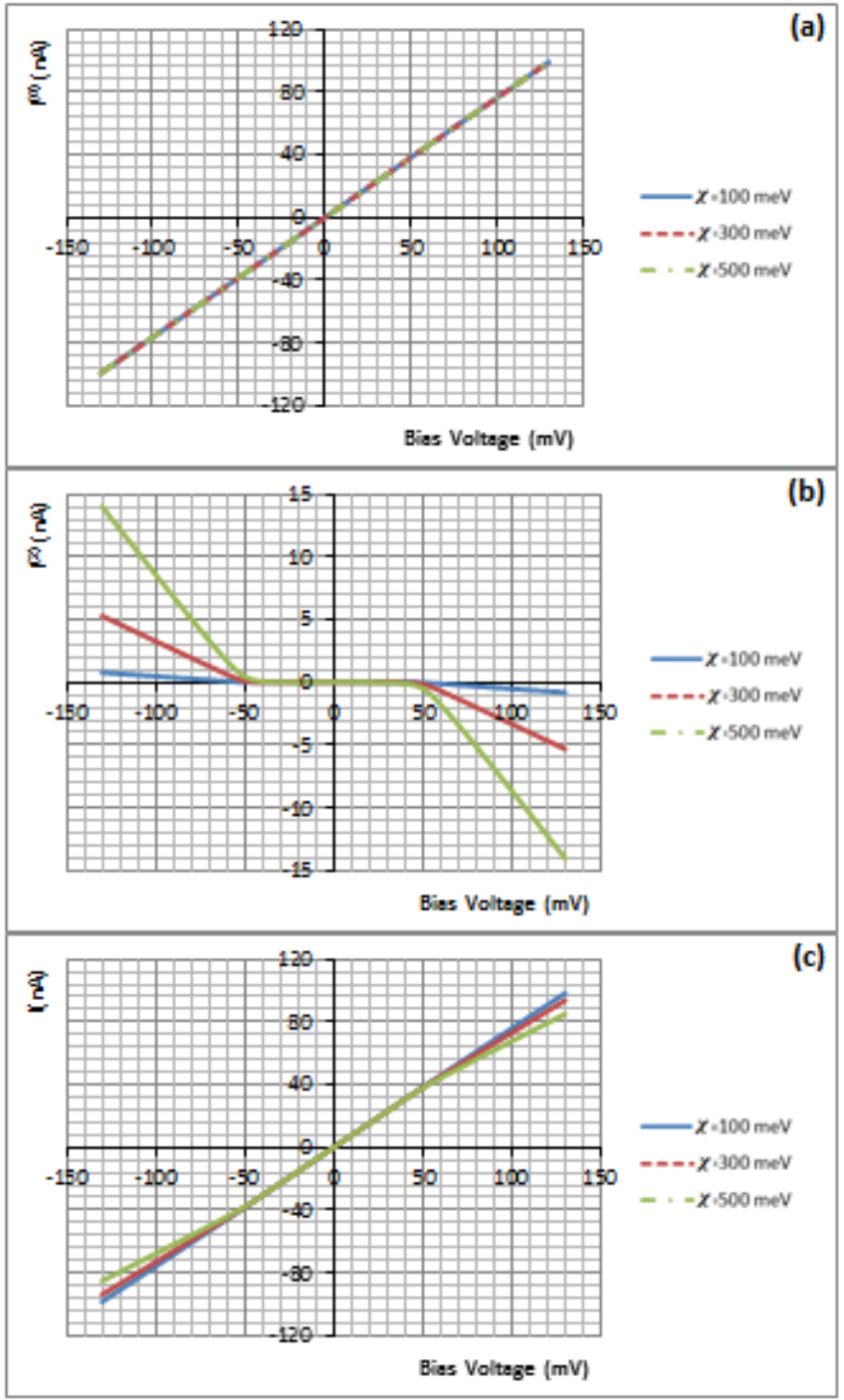

Fig. 1 : (a) The elastic current $\left(I_{\text {el }}^{(0)}\right)$ for $E_{\text {dot }}=$ $0.0 \mathrm{meV}, T=15 \mathrm{~K}, \Delta_{\mathrm{t}}=5 \mathrm{meV}, \Delta_{\mathrm{s}}=1000 \mathrm{meV}$, $\hbar \Omega=50 \mathrm{meV}$. (b) The tunneling current $\left(I^{(2)}\right)$. (c) The total tunneling cu+rrent $\left(I_{t}\right)$.
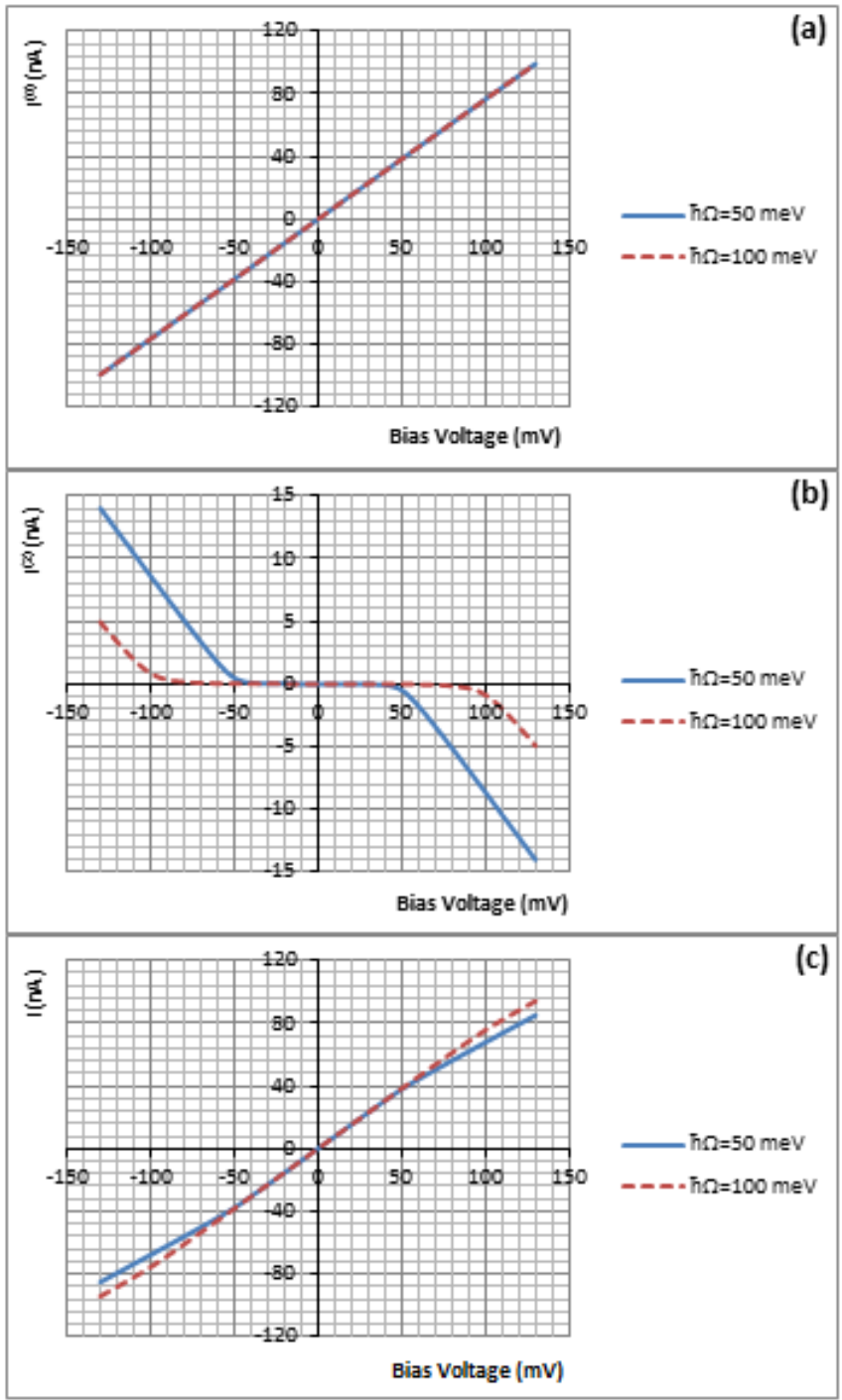

Fig. 2: (a) The elastic current $\left(\mathrm{I}_{\mathrm{el}}^{(0)}\right)$ for $\mathrm{E}_{\mathrm{dot}}=$ $0.0 \mathrm{meV}, \mathrm{T}=15 \mathrm{~K}, \Delta_{\mathrm{t}}=5 \mathrm{meV}, \Delta_{\mathrm{s}}=1000 \mathrm{meV}$, $\chi=500 \mathrm{meV}$. (b) The tunneling current $\left(I^{(2)}\right)$. (c) The total tunneling current $\left(I_{t}\right)$. 


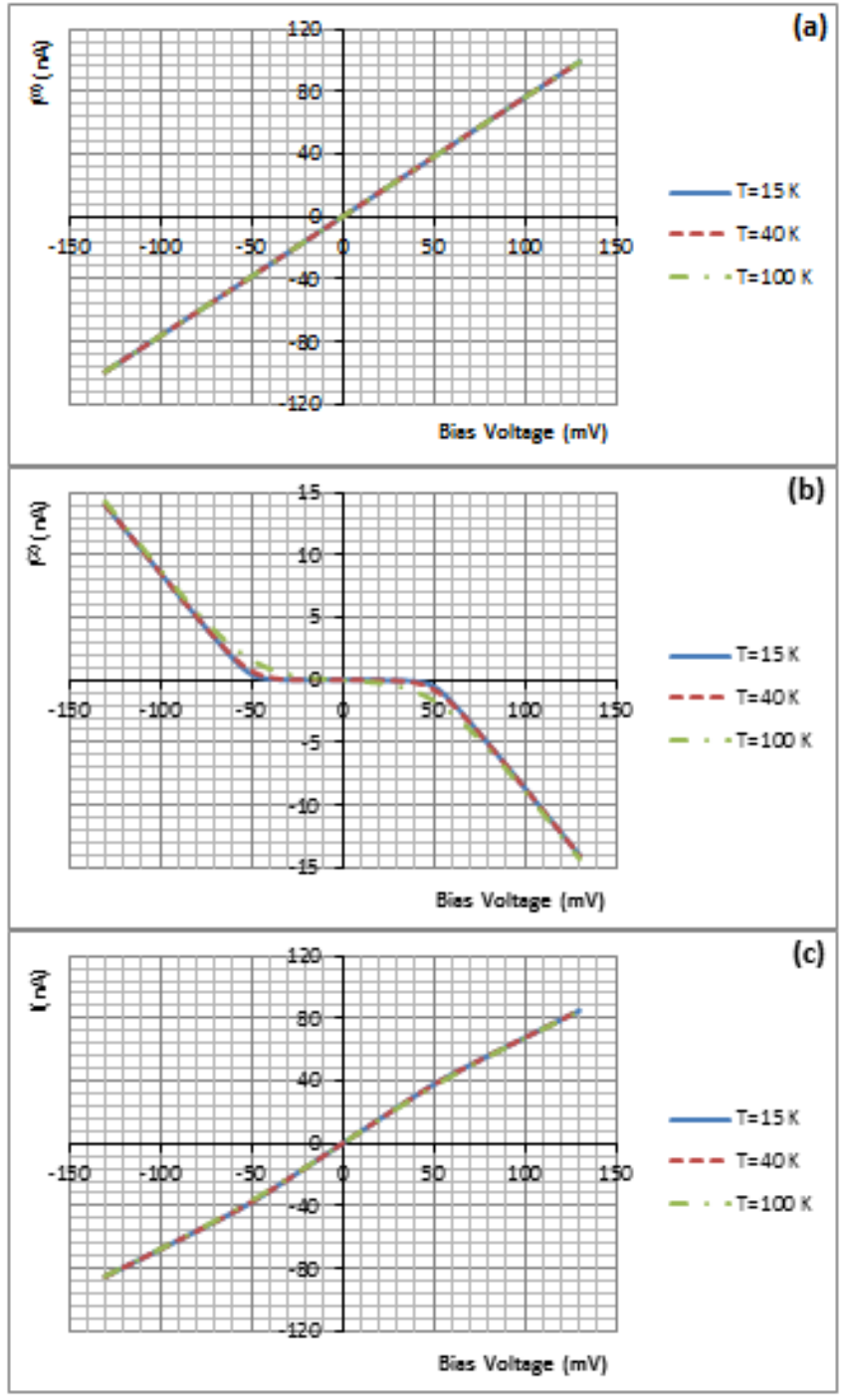

Fig. 3 : (a) The elastic current $\left(I_{e l}^{(0)}\right)$ for $E_{d o t}=$ $0.0 \mathrm{meV}, \hbar \Omega=50 \mathrm{meV}, \Delta_{\mathrm{t}}=5 \mathrm{meV}, \Delta_{\mathrm{s}}=1000 \mathrm{meV}$, $\chi=500 \mathrm{meV}$. (b) The tunneling current $\left(I^{(2)}\right)$. (c) The total tunneling current $\left(I_{t}\right)$.

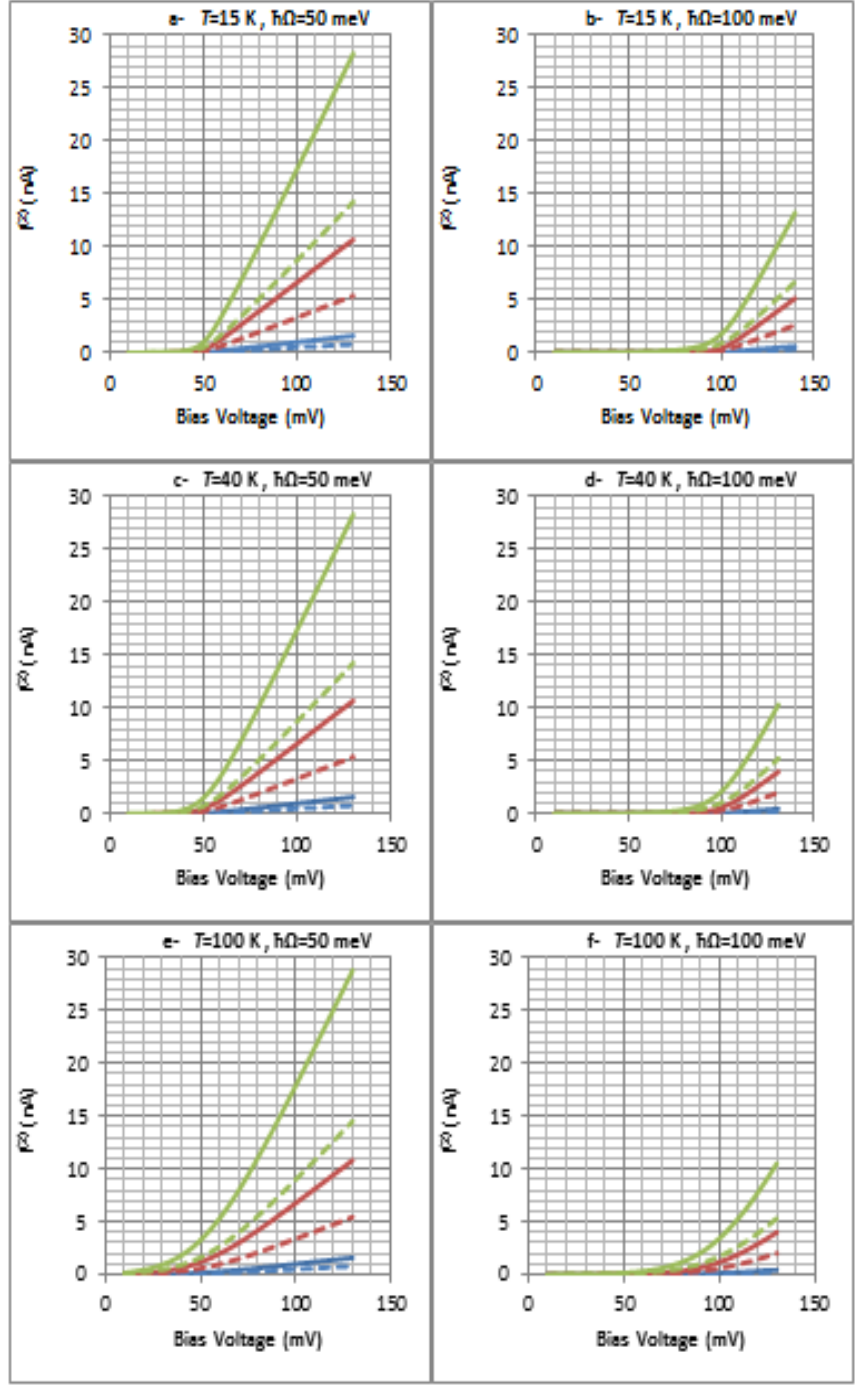

Fig. 4 : The elastic $\left(-I_{e l}^{(2)}\right.$, solid line) and inelastic $\left(I_{i n}^{(2)}\right.$ , dashed line) currents for $E_{\text {dot }}=0.0 \mathrm{meV}, \Delta_{\mathrm{t}}=$ $5 \mathrm{meV}, \Delta_{\mathrm{s}}=1000 \mathrm{meV}, \chi=100 \mathrm{meV} \quad, \chi=$ $300 \mathrm{meV} \quad, \chi=500 \mathrm{meV}$ 


\section{$\underline{\text { References }}$}

1. B. C. Stipe, M.A. Rezaei, and W. Ho, Science 280, 1732 (1998).

2. G. Binnig, N. Garcia, and H. Rohrer, Phys. Rev. B 32, 1336 (1985).

3. C. J. Chen, "Introduction to Scanning Tunneling Microscopy", New York, Oxford University Press, 2008.

4. B. C. Stipe, M. A. Rezaei, and W. Ho, Phys. Rev. Lett. 81, 1263 (1998).

5. H. J. Lee and W. Ho, Phys. Rev. Lett. 61, R16347 (2000).

6. L. Bartels, G. Meyer, K.-H. Rieder, D. Velic, E. Knoesel, A. Hotzel, M. Wolf, and G. Ertl, Phys. Rev. Lett. 80, 2004 (1998).

7. D. M. Eigler, C. P. Lutz, and W. E. Rudge, Nature 352, 600 (1991).

8. B. N. J. Persson and J. E. Demuth, Solid State Commun. 57, 769 (1986).

9. B. N. J. Persson and A. Baratoff, Phys. Rev. Lett. 59, 339 (1987).

10.C. Caroli, R. Combescot, P. Nozieres, and D. SaintJames, J. Phys. C 5, 21 (1972).

11.N. Mingo and K. Makoshi, Phys. Rev. Lett. 84, 3694 (2000).

12.N. Lorente and M. Persson, Phys. Rev. Lett. 85, 2997 (2000).

13.S. G. Tikhodeev, N. Mingo, K. Makoshi, T. Mii, and H. Ueba, Surf. Sci. 493, 63 (2000).

14.T. Mii, S.G. Tikhodeev, and H. Ueba, Surf. Sci. 502, 26 (2002).

15.T. Mii, S.G. Tikhodeev, and H. Ueba, Phys. Rev. B 68, 205406 (2003).
16.L. V. Keldysh and Zh. Eksp. Teor. Fiz. 47, 1515 (1964).

17.L. V. Keldysh, "Progress in Nonequilibrium Green's Functions II", World Scientific, Singapore, 2003.

18.S. G. Tikhodeev and H. Ueba, " Problems of Condensed Matter Physics: Quantum coherence phenomena in electron-hole and coupled matterlight systems", Oxford University Press, 2008.

19.H. Ueba, T. Mii, and S. G. Tikhodeev, Surf. Sci. 601, 5220 (2007).

20.T. Mii, S. G. Tikhodeev, and H. Ueba, Phys. Rev. B 68, 205406 (2003).

21.S. G. Tikhodeev and H. Ueba, Surf. Sci. 587, 25 (2005).

22.P. Hyldgaard, S. Hershfield, J.H. Davies, and J. W. Wilkins, Ann. Phys. 236, 1 (1994).

23.C. Delerue and M. Lannoo, "Nanostructures: theory and modeling", springer, 2004. 